\title{
High-sensitivity C-reactive protein and exercise-induced changes in subjects suspected of coronary artery disease
}

This article was published in the following Dove Press journal:

Journal of Inflammation Research

22 March 2014

Number of times this article has been viewed

\author{
Mette Rauhe Mouridsen' \\ Olav Wendelboe Nielsen' \\ Christian Malchau Carlsen' \\ Nick Mattsson' \\ Martin H Ruwald ${ }^{2}$ \\ Zeynep Binici ${ }^{3}$ \\ Ahmad Sajadieh' \\ 'Department of Cardiology, \\ Copenhagen University Hospital \\ of Bispebjerg, Copenhagen NV, \\ Denmark; ${ }^{2}$ Department of Cardiology, \\ Gentofte University Hospital, \\ Hellerup, Denmark; ${ }^{3}$ Department of \\ Cardiology, Herlev Hospital, Herlev, \\ Denmark
}

Background: Inflammation plays a major role in the development of atherosclerosis. We wanted to investigate the effects of exercise on high-sensitivity (hs) C-reactive protein (CRP) in subjects who were suspected of having coronary artery disease (CAD).

Methods: Blood samples were obtained before, 5 minutes after, and 20 hours after an exercise test in 155 subjects who were suspected of CAD. Coronary anatomy was evaluated by computed tomography coronary angiography and/or coronary angiography.

Results: Median baseline hs-CRP was higher in subjects with $\geq 50 \%$ coronary artery lumen diameter stenosis $(n=41)$, compared with non-CAD-subjects $(n=114), 2.93 \mathrm{mg} / \mathrm{L}$ (interquartile range $1.03-5.06 \mathrm{mg} / \mathrm{L}$ ) and $1.30 \mathrm{mg} / \mathrm{L}$ (interquartile range $0.76-2.74 \mathrm{mg} / \mathrm{L}$ ), respectively, $P=0.007$. In multivariate analyses testing conventional risk factors, hs-CRP proved borderline significant, odds ratio $=2.32, P=0.065$. Adding baseline hs-CRP to the results of the exercise test did not improve the diagnostic evaluation. Baseline natural logarithm (Ln) hs-CRP was positively associated with body mass index and baseline Ln-transformed hs troponin T levels, and negatively associated with the daily life activity level. An increase in hs-CRP of $0.13 \mathrm{mg} / \mathrm{L}$ (interquartile range $0.05-0.24 \mathrm{mg} / \mathrm{L}$ ) from baseline to 5 minutes after peak exercise was found $(P<0.0001)$, but the increase was not associated with presence of CAD. From baseline to 20 hours after exercise, no increase in hs-CRP was found.

Conclusion: In conclusion, hs-CRP was not independently associated with CAD. Hs-CRP increased immediately as a response to the exercise, and the increase was modest and not associated with CAD. The results indicate that exercise has potential to cause unwanted variations in hs-CRP and that exercise prior to hs-CRP measurements in subjects included in epidemiological studies, therefore, should be avoided.

Keywords: inflammation, stable angina pectoris, atherosclerosis, exercise testing

\section{Introduction}

The Framingham Heart Study invented the term "coronary risk factors" when - back in the $1950 \mathrm{~s}$ - they reported that hypercholesterolemia and hypertension were associated with an increased risk of coronary artery disease (CAD). ${ }^{1}$ Soon hereafter, other risk factors were recognized as contributing to the development and progression of atherosclerosis and to its manifestations. ${ }^{2-6}$ For many years, hypercholesterolemia, hypertension, smoking, insulin resistance, mental stress, and physical inactivity were believed to be the sole reasons for the development of CAD and subsequent cardiovascular disease. Modification of risk factors was expected to eliminate CAD by the end of the 20th century. However, because of the fact that a considerable proportion $(20 \%)$ of those subjects with cardiovascular events were found to have no known risk 
factors, it became more and more obvious that atherosclerosis was not solely a lipid-based disorder. ${ }^{7-9}$

Great attention toward the inflammatory marker C-reactive protein (CRP) arose when the Justification for the Use of statins in Primary prevention: an Intervention Trial Evaluating Rosuvastatin (JUPITER) trial reported beneficial effects of statin therapy in subjects with normal low density lipoprotein (LDL) cholesterol levels and elevated CRP levels. ${ }^{10}$ Numerous studies followed reporting similar results, suggesting that the anti-inflammatory effect of statins reduces cardiovascular events. ${ }^{11,12}$ Drug therapies reducing low-density lipoprotein (LDL) cholesterol, and not CRP, such as postmenopausal hormone replacement therapy, failed to reduce cardiovascular risk in randomized trials. ${ }^{13}$

Whether CRP contributes causally to the development of CAD or whether it is just a surrogate marker of underlying chronic inflammation and generalized atherosclerosis is controversial. Four large Mendelian studies have reported that genetic variations in the $C R P$ gene, associated with increased CRP levels, were not associated with an increased risk of CAD. ${ }^{14}$ These results question the causality between CRP and CAD and indicate that an elevated CRP is a marker for the extent of atherosclerosis, the inflammatory activity, and the vulnerability of atherosclerotic plaques without playing an active role in the process.

Studies have demonstrated CRP elevations immediately after an acute myocardial infarction, indicating that the protein might also react as an acute marker of destabilized atherosclerotic plaques. ${ }^{15,16}$ It remains to be uncovered whether it is a dynamic biomarker synthesized and released as a response to minor episodes of reversible myocardial ischemia.

We aimed to investigate the effects of exercise on highsensitivity (hs) CRP levels in subjects suspected of CAD.

\section{Materials and methods \\ Study population}

Between August 2009 and June 2011, a total of 601 consecutive subjects were screened. A total of 316 subjects with chest complaints referred to ambulatory evaluation at the Department of Cardiology at the Copenhagen University Hospital of Bispebjerg in Denmark were enrolled in this prospective observational cohort study. In total, 72 subjects were not able to meet 20 hours after the exercise test (ET) to have blood samples taken, and 87 subjects were not thought to have CAD when evaluated by an experienced study physician. These 159 subjects were excluded from the statistical analyses, resulting in a total of 157 study subjects. Two subjects were excluded at a later stage due to very high baseline levels of hs-CRP $(31.34 \mathrm{mg} / \mathrm{L}$ and $67.94 \mathrm{mg} / \mathrm{L})$, indicating an acute bacterial infection. Figure 1 illustrates the inclusion process.

Inclusion criteria were: age $\geq 18$ years; symptoms of ischemic heart disease; and ability to provide written, informed consent. Exclusion criteria were: renal insufficiency (serum creatinine $>145 \mu \mathrm{mol} / \mathrm{L}$ ); a history of myocardial infarction; significant valvular disease; pacemaker device; permanent atrial fibrillation; left bundle branch block; history of heart failure with a left ventricular ejection fraction $\leq 45 \%$; prior percutaneous coronary intervention, or coronary artery bypass grafting; and active cancer disease. The Regional Ethics Committee in the Capital Region of Denmark and the Danish Data Protection Agency approved the study protocol. Written, informed consent was obtained from all study subjects.

\section{Definitions of variables}

For the purpose of this study, hypertension was defined as known hypertension, or a systolic blood pressure $\geq 160 \mathrm{mmHg}$ at the screening visit, measured twice after at least 10 minutes of rest. Diabetes mellitus was defined by self-reported history, or antidiabetic drugs and/or a fasting blood glucose $>126 \mathrm{mg} / \mathrm{dL}(7.0 \mathrm{mmol} / \mathrm{L})$. Tobacco use was selfreported and defined as positive if the patient was an active smoker or a former smoker with a history of more than 3 years of smoking. A family history of early CAD was defined as $\geq$ one first-degree relative with clinically significant CAD or sudden death presenting before the age of 60 . Typical/ atypical angina was defined as substernal chest discomfort of characteristic quality and duration, provoked by exertion or emotional stress, and relieved by rest and/or nitroglycerin (with typical angina meeting all the criteria and atypical angina meeting two of the criteria). Noncardiac chest pain met one or none of the characteristics. ${ }^{17}$

Echocardiography was performed with determination of left ventricular ejection fraction and wall thickness using two-dimensional echo images.

\section{Exercise test protocol}

Study subjects were classified according to the pretest probability of CAD (0\%-100\%), using an updated DiamondForrester score that was based on age, sex, and symptoms. ${ }^{18,19}$ Subjects were classified as having low risk $(<20 \%$ probability of CAD), intermediate risk $(20 \%-80 \%)$, or high risk $(>80 \%)$. Self-reported daily life activity level within the prior 3 months was registered. Subjects were then grouped into four groups: 1) $<1$ hour of physical activity/week; 2) 1-3 hours of physical 


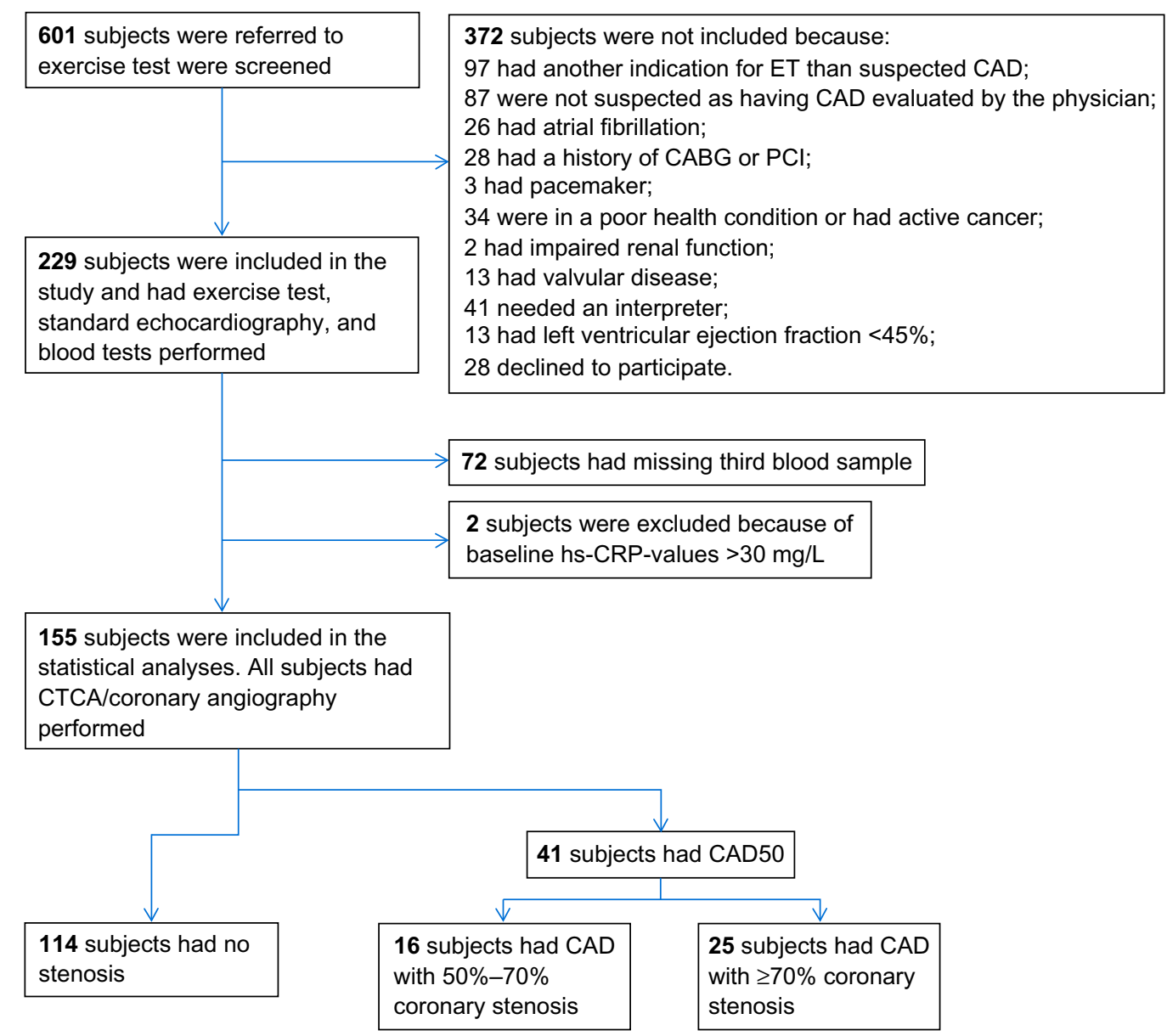

Figure I Flow diagram illustrating the inclusion process.

Note: CAD50 refers to subjects with $\geq 50 \%$ coronary artery lumen diameter stenosis.

Abbreviations: $\mathrm{ET}$, exercise test; $\mathrm{CAD}$, coronary artery disease; $\mathrm{CABG}$, coronary artery bypass grafting; PCl, percutaneous coronary intervention; hs-CRP, high-sensitivity C-reactive protein; CTCA, computed tomography coronary angiography.

activity/week; 3) 3-6 hours of physical activity/week; and 4) $>6$ hours of physical activity/week.

All study subjects underwent a symptom-limited multistage bicycle ET. To reach a recommended optimal exercise time between 6 and 12 minutes, the exercise test protocol was either 25 watts/25 watts/2 minutes (meaning a start workload at 25 watts with an increase in workload of 25 watts every 2 minutes) or 50 watts $/ 50$ watts $/ 2$ minutes with respect to the subjects' age and physical condition. Resting heart rate, blood pressure, and 12-lead electrocardiography (ECG) were recorded automatically before exercise. A 12-lead ECG was continuously recorded during exercise and recovery. Blood pressure was reported automatically every 2 minutes during the exercise and recovery. The ST segment deviation was assessed automatically and visually controlled and interpreted by an experienced cardiologist. The workload was expressed in metabolic equivalents (METs). The criteria for test interruption by the physician were ST segment elevation $>0.1 \mathrm{mV}$, ventricular arrhythmias, or a drop in blood pressure $>20 \mathrm{mmHg}$. The criteria for test interruption by the patient were extreme fatigue, dyspnea, dizziness, or exercise-limiting angina pectoris. The ET was considered positive (typical for myocardial ischemia) if at least one of the following two criteria occurred: 1) horizontal/downsloping ST segment depression $\geq 0.1 \mathrm{mV}$ in two neighboring leads; or 2) chest pain typical of angina pectoris. ${ }^{20}$ A negative test was defined as a test where none of the criteria for a positive test occurred and where a peak heart rate of at least $85 \%$ of predicted maximum heart rate (220 minus age) was reached. The ETs that were neither positive nor negative were defined as inconclusive.

\section{Evaluation of the coronary arteries}

The coronary anatomy was evaluated in all subjects. Subjects with a positive ET were referred directly to coronary angiography, while subjects with an inconclusive or negative ET were referred to computed tomography coronary 
angiography (CTCA). If the CTCA clearly ruled out coronary stenosis, then no further testing was performed, while subjects with a definite or possible luminal diameter stenosis of $\geq 50 \%$ were referred to coronary angiography.

The CTCAs were performed using a 64-slice multidetector scanner (Brilliance CT 64, Philips Medical Systems, Best, the Netherlands) with prospective ECG gating and image acquisition in the mid-diastolic phase $(70 \%-80 \%$ of $\mathrm{R}$ wave to $\mathrm{R}$ wave interval). An oral $\beta$-blocker (metoprolol, 50-100 mg) was used to lower the heart rate. The coronary calcium score was measured in a noncontrast-enhanced scan. A contrastenhanced scan was performed with a bolus contrast medium (Visipaque $^{\mathrm{TM}} 320 \mathrm{I} / \mathrm{mL}$, GE Healthcare AS, Oslo, Norway) injected in an antecubital vein with a flow rate of $5 \mathrm{~mL} / \mathrm{sec}$ ond, followed by $50 \mathrm{~mL}$ saline. An automatic triggering of the contrast medium injection with a region of interest at the descending aorta (threshold of $120 \mathrm{HU}$ ) was used. Scanning was performed at $120 \mathrm{kV}$; effective tube current, 600-1,000 $\mathrm{mA}$; slice collimation, $64 \times 0.625 \mathrm{~mm} ; 0.4$ seconds, gantry rotation time; and pitch, 0.2 .

An analysis of the coronary arteries was performed using a segmental evaluation of each coronary artery, according to the American Heart Association classification using a 15-segment model. ${ }^{21}$ Two experienced readers did the evaluations of the images. Plaques and regions with lumen narrowing were marked, and the patients were grouped according to the degree of CAD: 1) no calcifications or stenosis (Agatston score $=0$ and no stenosis) 2 ) calcifications without stenosis (Agatston score $>0$ and no stenosis or $<50 \%$ lumen diameter stenosis); and 3 ) suspected stenosis $\geq 50 \%$.

The coronary angiographies were performed using a commonly performed technique. Two experienced angiographers evaluated the coronary angiographies. All diagnoses were based on consensus with an experienced percutaneous interventionalist from a tertiary center and subjects with stenosis of uncertain significance went to the tertiary center for fractional flow reserve measurement.

Subjects were grouped in the following groups, according to the degree of CAD: 1) no calcifications; 2) calcifications without stenosis; 3) CAD with 50\%-70\% stenosis; and 4) CAD with $\geq 70 \%$ stenosis. Dichotomous variables were created representing $\geq 50 \%$ lumen diameter stenosis (CAD50) and $\geq 70 \%$ lumen diameter stenosis (CAD70).

\section{hs-CRP and hs troponin T measurements}

The blood samples were obtained in the antecubital vein with the subjects in a sitting position before the ET, 5 minutes after peak exercise, and again 20 hours after peak exercise. The blood samples were obtained in clot activator serum tubes and placed at room temperature for 30 minutes and centrifuged for 10 minutes at 3,500 rpm. Serum was immediately thereafter aspirated and stored at $-80^{\circ} \mathrm{C}$.

Five months after the inclusion period had stopped, the stored samples were analyzed for hs-CRP and high-sensitivity troponin $\mathrm{T}$ (hs-TnT). The hs-CRP was measured using a high-sensitivity immunoturbidimetric assay on the Hitachi autoanalyzer (Roche Diagnostics Corporation, Indianapolis, IN, USA). The coefficient of variation obtained from blinded simultaneous analyzed quality controls was $<3 \%$. The detection range was $0.15-20.0 \mathrm{mg} / \mathrm{L}$. The hs-TnT measurements were performed using a high-sensitivity assay (Elecsys hs Troponin T, Roche Diagnostics). The limit of blank was $3.00 \mathrm{pg} / \mathrm{mL}$, the assay's $10 \%$ coefficient of variance threshold was $13 \mathrm{pg} / \mathrm{mL}$, and the 99th percentile value in healthy subjects was $14 \mathrm{pg} / \mathrm{mL}$.

All biochemical testing was performed by study personnel blinded to, and with no access to, each participant's files.

\section{Statistical analysis}

The baseline characteristics are reported as percentages for categorical variables and means and standard deviations (SDs) for normally distributed continuous variables, while medians and interquartile ranges (IQR) for non-normally distributed continuous variables are reported. We compared continuous variables using the Student's $t$-test or Wilcoxon rank-sum test as appropriate. The receiver operating characteristic curves indicated an optimal cut-off value for baseline hs-CRP in diagnosing CAD at $2.93 \mathrm{mg} / \mathrm{L}$. A dichotomized variable defining increased hs-CRP $>2.93 \mathrm{mg} / \mathrm{L}$ was created. Multivariate linear regression analyses with natural logarithm (Ln)-transformed baseline hs-CRP and delta-hsCRP as dependent variables were performed to test associations with the known cardiovascular risk factors, presence of CAD and ET parameters. Multivariate logistic regression analyses using CAD50 as the response variable were performed. The covariates' age, sex, estimated glomerular filtration rate, smoking, diabetes, cholesterol, left ventricular ejection fraction, left ventricular hypertrophy, systolic blood pressure, body mass index (BMI), waist circumference, waist-hip ratio, daily physical activity level, and hs-CRP were tested in the model. Covariates with a $P$-value $<0.100$ were kept in the model. Linear regression analyses were used to evaluate possible associations between Ln hs-CRP/delta$\mathrm{CRP}$ and conventional risk factors, exercise test parameters, and the Agatston score. A $P$-value $<0.05$ was regarded as statistically significant. Statistical analyses and graphics 
were performed using STATA for Windows, version 11.0 (StataCorp LP, College Station, TX, USA).

\section{Results}

\section{Baseline characteristics, exercise testing, and evaluation of coronary arteries}

Figure 1 shows the inclusion process where the overall prevalence of CAD50 was $26 \%$ (41/155). Patients with CAD50 were older, more often male, with a higher prevalence of diabetes and hypertension (Table 1).

In total, six subjects with CAD70 received optimal medical therapy instead of revascularization because their lesions were not technically suitable for percutaneous coronary intervention $(n=3)$, presumed satisfactory effect of optimal medical therapy $(\mathrm{n}=2)$, and an unsuccessful attempt to revascularize, resulting in minor dissection $(\mathrm{n}=1)$.

Table 2 shows ET results in all study subjects and in nonCAD versus CAD50 subjects.

\section{Baseline hs-CRP and conventional risk factors}

In multivariate linear regression analyses, baseline Ln-hs-CRP was positively associated with BMI and baseline Ln-hs-TnT levels. It was negatively associated with the daily life activity level (Table 3). Figure 2 is three box plots that show median hs-CRP levels in subjects with hs-TnT $\leq 3.00 \mathrm{pg} / \mathrm{mL}$ ( $\mathrm{n}=79$ ), subjects with hs-TnT levels between 3.00 and $7.26 \mathrm{pg} / \mathrm{mL}$ $(\mathrm{n}=38)$, and subjects with very high hs-TnT levels $>7.26$ $\mathrm{pg} / \mathrm{mL}(\mathrm{n}=38)$.

\section{Baseline hs-CRP and Agatston score}

In univariate linear regression analysis, baseline Ln-hs-CRP was positively associated with the Agatston score on CTCA, coefficient $=0.0005, P=0.035$. However, when including conventional risk factors (age, sex, and diabetes), the association was no longer significant (data not shown).

\section{Baseline hs-CRP and CAD}

Median baseline hs-CRP was significantly higher in subjects with CAD50 when compared to non-CAD subjects, 2.93 $\mathrm{mg} / \mathrm{L}$ (IQR $1.03-5.06 \mathrm{mg} / \mathrm{L}$ ) and $1.30 \mathrm{mg} / \mathrm{L}$ (IQR 0.76-2.74 $\mathrm{mg} / \mathrm{L}$ ), respectively, $P=0.007$ (Table 1 and Figure 3). Generating receiver operating characteristic curves, an area under the curve (AUC) of 0.642 was found (Figure 4), and an optimal cut-off point for diagnosing CAD50 of about 2.93 $\mathrm{mg} / \mathrm{L}$ was found. With a cut-off point at $2.93 \mathrm{mg} / \mathrm{L}$, a sensitivity of $51.2 \%$ and a specificity of $78.1 \%$ were achieved. In univariate logistic regression analysis with CAD50 as the outcome variable, hs-CRP $>2.93 \mathrm{mg} / \mathrm{L}$ resulted in odds

Table I Baseline variables in all study subjects and divided in non-CAD and CAD50 subjects

\begin{tabular}{|c|c|c|c|c|}
\hline Variables & $\begin{array}{l}\text { All subjects } \\
n=I 55\end{array}$ & $\begin{array}{l}\text { Non-CAD } \\
n=1 \mid 4\end{array}$ & $\begin{array}{l}\text { CAD50 } \\
n=4 I\end{array}$ & $P$-value \\
\hline Age, years, mean $\pm S D$ & $58.3 \pm 11.7$ & $55.6 \pm 11.2$ & $65.9 \pm 9.6$ & $<0.0001$ \\
\hline Male sex, n (\%) & $95(6 \mid .3)$ & $61(53.5)$ & $34(82.9)$ & 0.001 \\
\hline $\mathrm{BMI}, \mathrm{kg} / \mathrm{m}^{2} \pm \mathrm{SD}$ & $26.9 \pm 4.2$ & $26.7 \pm 4.4$ & $27.3 \pm 3.9$ & NS \\
\hline Waist circumference, $\mathrm{cm}$, mean $\pm \mathrm{SD}$ & $98.5 \pm 12.4$ & $97.1 \pm 12.2$ & $102.2 \pm 12.4$ & $<0.05$ \\
\hline Waist-hip ratio, mean $\pm S D$ & $0.93 \pm 0.08$ & $0.92 \pm 0.08$ & $0.98 \pm 0.07$ & 0.0001 \\
\hline Hs-CRP, mg/L, median (IQR) & $\mathrm{I} .53(0.83-3.57)$ & $\mathrm{I} .30(0.76-2.74)$ & $2.93(1.03-5.06)$ & 0.007 \\
\hline Delta hs-CRP, median (IQR) & $0.13(0.05-0.24)$ & $0.12(0.05-0.24)$ & $0.17(0.07-0.29)$ & NS \\
\hline \multicolumn{5}{|l|}{ Risk factors } \\
\hline Diabetes, n (\%) & I7 (I I.0) & $8(7.0)$ & $9(22.0)$ & 0.02 \\
\hline Former or current smoker, n (\%) & I I 3 (72.9) & $83(72.8)$ & $30(73.2)$ & NS \\
\hline Hypercholesterolemia, n (\%) & $57(36.8)$ & $38(33.3)$ & $19(46.3)$ & NS \\
\hline Hypertension, n (\%) & $72(46.5)$ & $46(40.4)$ & $26(63.4)$ & 0.02 \\
\hline Family history of early CAD, n (\%) & $43(27.7)$ & $27(23.7)$ & $16(39.0)$ & NS \\
\hline \multicolumn{5}{|l|}{ Symptoms } \\
\hline Typical/atypical angina, n (\%) & $38(24.5)$ & I3 (I I.4) & $25(61.0)$ & $<0.0001$ \\
\hline Noncardiac chest pain, n (\%) & I I 7 (75.5) & I0I (88.6) & $16(39.0)$ & $<0.0001$ \\
\hline \multicolumn{5}{|l|}{ Medication } \\
\hline Aspirin, n (\%) & $52(35.1)$ & $27(25.2)$ & $25(61.0)$ & $<0.0001$ \\
\hline$\beta$-blockers, n (\%) & $25(16.9)$ & $17(15.9)$ & $8(19.5)$ & NS \\
\hline Nitrates, n (\%) & $5(3.4)$ & $2(1.9)$ & $3(7.3)$ & NS \\
\hline Calcium antagonists, n (\%) & $28(18.9)$ & $16(15.0)$ & $12(29.3)$ & NS \\
\hline
\end{tabular}

Notes: Data are presented as mean \pm SD, median (IQR), or $n$ (\%). CAD50 refers to subjects with $\geq 50 \%$ coronary artery lumen diameter stenosis.

Abbreviations: CAD, coronary artery disease; SD, standard deviation; BMI, body mass index; NS, nonsignificant; hs-CRP, high-sensitivity C-reactive protein; IQR, interquartile range. 
Table 2 Stress test results in all study subjects and divided in non-CAD and CAD50 subjects

\begin{tabular}{|c|c|c|c|c|}
\hline & $\begin{array}{l}\text { All subjects } \\
n=\mid 55\end{array}$ & $\begin{array}{l}\text { Non-CAD } \\
n=1 / 4\end{array}$ & $\begin{array}{l}\text { CAD50 } \\
n=4 !\end{array}$ & $P$-value \\
\hline Pretest probability, $\% \pm S D$ & $37.0 \pm 25.2$ & $28.1 \pm 19.5$ & $61.7 \pm 22.8$ & $<0.000$ I \\
\hline \multicolumn{5}{|l|}{ Stress test performance } \\
\hline Duration, minutes $\pm S D$ & $9.3 \pm 3.7$ & $9.3 \pm 3.7$ & $9.3 \pm 3.4$ & NS \\
\hline Maximum workload, METs \pm SD & $8.4 \pm 2.9$ & $8.9 \pm 3.0$ & $7.2 \pm 1.9$ & 0.001 \\
\hline Peak HR, beats/minute \pm SD & $149.3 \pm 22.1$ & $152.6 \pm 22.0$ & $140.1 \pm 19.9$ & 0.002 \\
\hline$\%$ of max predicted $\mathrm{HR}, \% \pm \mathrm{SD}$ & $92.0 \pm 11.2$ & $92.6 \pm 11.2$ & $90.5 \pm 11.3$ & NS \\
\hline Subjects reaching $85 \%$ of $\max$ & $123(79.4)$ & $93(81.6)$ & $30(73.2)$ & NS \\
\hline \multicolumn{5}{|l|}{ predicted HR, n (\%) } \\
\hline Peak SBP, mmHg \pm SD & $193.8 \pm 30.9$ & $192.7 \pm 30.9$ & $196.9 \pm 28.4$ & NS \\
\hline \multicolumn{5}{|l|}{ Reason for termination of test } \\
\hline Exhaustion, n (\%) & $104(67.1)$ & $76(66.7)$ & $28(68.3)$ & NS \\
\hline Angina, $\mathrm{n}(\%)$ & $4(2.6)$ & $0(0)$ & $4(9.8)$ & $<0.001$ \\
\hline Muscular weakness, n (\%) & $23(14.8)$ & $18(15.8)$ & $5(12.2)$ & NS \\
\hline Dyspnea, n (\%) & $20(12.9)$ & $17(14.9)$ & $3(7.3)$ & NS \\
\hline Arrhythmia, n (\%) & $4(2.6)$ & $3(2.6)$ & $\mathrm{I}(2.4)$ & NS \\
\hline Angina & & & & $<0.0001$ \\
\hline No, n (\%) & 127 (8I.9) & $103(90.4)$ & $24(58.5)$ & \\
\hline Nonlimiting, n (\%) & $24(15.5)$ & II (9.7) & $13(3 \mid .7)$ & \\
\hline Limiting, n (\%) & $4(2.6)$ & $0(0.0)$ & $4(9.8)$ & \\
\hline ST depression $(\mathrm{mV})$ & & & & 0.10 \\
\hline $0.0, \mathrm{n}(\%)$ & II 4 (73.6) & $88(77.2)$ & $26(63.4)$ & \\
\hline$\geq 1.0, \mathrm{n}(\%)$ & $4 \mid(26.5)$ & $26(22.8)$ & $15(36.6)$ & \\
\hline Conclusion of the ET & & & & 0.04 \\
\hline Conclusive negative ET, n (\%) & $88(56.1)$ & $7 \mid(6 I .2)$ & $17(4 \mid .5)$ & \\
\hline Inconclusive ET, n (\%) & $22(14.2)$ & $16(14.0)$ & $6(14.6)$ & \\
\hline Positive ET, n (\%) & $45(28.7)$ & $27(23.3)$ & $18(43.9)$ & \\
\hline ET protocol used & & & & $<0.001$ \\
\hline 25 watts $/ 25$ watts $/ 2$ minutes, $\mathrm{n}(\%)$ & $119(76.8)$ & $79(69.3)$ & $40(97.6)$ & \\
\hline 50 watts $/ 50$ watts $/ 2$ minutes, $\mathrm{n}(\%)$ & $36(23.2)$ & $35(30.7)$ & I $(2.4)$ & \\
\hline
\end{tabular}

Notes: Data are presented as mean \pm SD, or $n$ (\%). CAD50 refers to subjects with $\geq 50 \%$ coronary artery lumen diameter stenosis.

Abbreviations: CAD, coronary artery disease; SD, standard deviation; MET, metabolic equivalent; HR, heart rate; SBP, systolic blood pressure at rest; ET, exercise test.

ratio $(\mathrm{OR})=3.7, P=0.001$. However, when adjusting for other significant risk factors found by multivariate logistic regression analysis, high age, male sex, and diabetes were more strongly associated with CAD50 than hs-CRP, which (in the adjusted model) proved only borderline significant (Table 4).

In subjects with CAD70, the hs-CRP levels were significantly higher than in subjects without CAD70, $2.98 \mathrm{mg} / \mathrm{L}$ and $1.36 \mathrm{mg} / \mathrm{L}$, respectively, $P=0.03$. If a

Table 3 Multivariate linear regression analysis with Ln hs-CRP as dependent variable

\begin{tabular}{llll}
\hline & \multicolumn{2}{l}{ Outcome Ln hs-CRP } \\
\cline { 2 - 4 } & Coefficient & $\mathbf{9 5 \%} \mathbf{~ C l}$ & P-value \\
\hline Daily life activity level & -0.167 & -0.31 I to -0.023 & 0.023 \\
BMI & 0.047 & $0.011-0.082$ & 0.010 \\
Ln hs-TnT & 0.513 & $0.306-0.721$ & $<0.001$ \\
\hline
\end{tabular}

Notes: Cholesterol, cigarette smoking, diabetes, sex, age, left ventricular ejection fraction, estimated glomerular filtration rate, waist circumference, waist-hip ratio, and CAD50/CAD70 were tested in the model but excluded because $P$-value $>0.1$. Abbreviations: Ln, natural logarithm; hs, high-sensitivity; CRP, C-reactive protein; $\mathrm{Cl}$, confidence interval; BMI, body mass index; $\mathrm{TnT}$, troponin $\mathrm{T}$. cut-off point for diagnosing CAD70 of $2.98 \mathrm{mg} / \mathrm{L}$ was used, an $\mathrm{AUC}=0.641$ was found. In univariate logistic regression analysis with CAD70 as outcome variable, hs-CRP $>2.98 \mathrm{mg} / \mathrm{L}$ resulted in an $\mathrm{OR}=3.3, P=0.008$. However, adjusting for age, sex, and diabetes in multivariate logistic regression analysis rendered the hs-CRP insignificant.

Figure 5 illustrates the median hs-CRP values in study subjects grouped in four groups: no calcification in coronary arteries $(n=64)$; calcifications without stenosis $(n=50)$; CAD with $50 \%-70 \%$ stenosis $(\mathrm{n}=16)$; and CAD with $\geq 70 \%$ stenosis $(n=25)$. Using CAD as an ordinal variable, hs-CRP increased with an increasing degree of CAD (Figure 5), but the association disappeared when adjusting for conventional risk factors (data not shown).

\section{Delta hs-CRP and CAD}

A statistically significant median increase in hs-CRP of $0.13 \mathrm{mg} / \mathrm{L}$ (IQR $0.05-0.24 \mathrm{mg} / \mathrm{L}$ ) from baseline to 5 minutes after peak exercise for the entire study group 


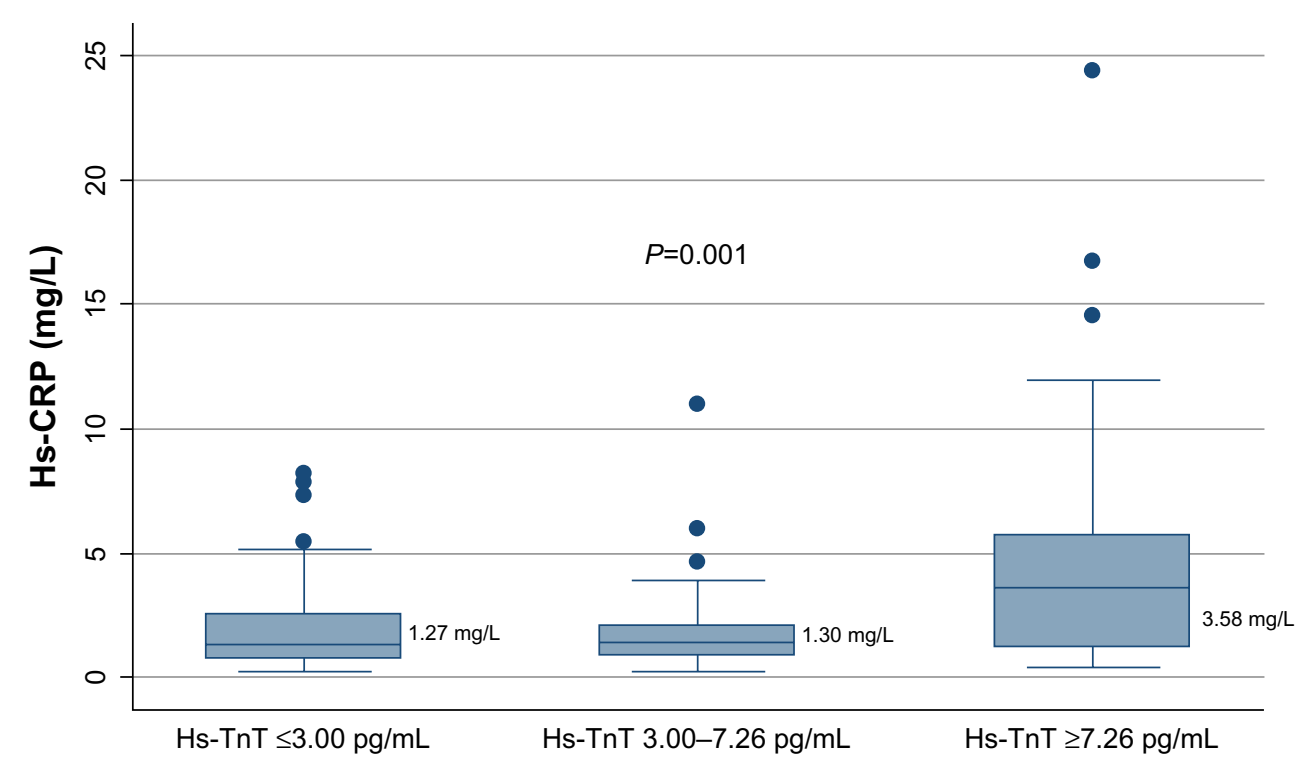

Figure 2 Box plots showing median hs-CRP levels.

Notes: Shown are subjects with hs- $\mathrm{TnT} \leq 3.00 \mathrm{pg} / \mathrm{mL}(\mathrm{n}=79$ ), subjects with hs-TnT levels between 3.00 and $7.26 \mathrm{pg} / \mathrm{mL}$ ( $\mathrm{n}=38$ ), and subjects with very high hs-TnT levels $>7.26 \mathrm{pg} / \mathrm{mL}(\mathrm{n}=38)$.

Abbreviations: hs, high-sensitivity; CRP, C-reactive protein; TnT, troponin T.

was seen, $P<0.0001$. However, the exercise-induced increase in hs-CRP was not significantly associated with presence of CAD50 in that CAD50 subjects had a median increase of $0.17 \mathrm{mg} / \mathrm{L}$ (IQR $0.07-0.29 \mathrm{mg} / \mathrm{L}$ ) while non-CAD subjects had an increase of $0.12 \mathrm{mg} / \mathrm{L}$ (IQR $0.05-0.24 \mathrm{mg} / \mathrm{L}), P=0.139$. Similar results were found evaluating CAD70. Neither was the increase in hs-CRP associated with any of the ET parameters (exercise time, METs, ST segment depression, angina during the ET, increase in systolic blood pressure, increase in heart rate, and increase in hs-TnT).

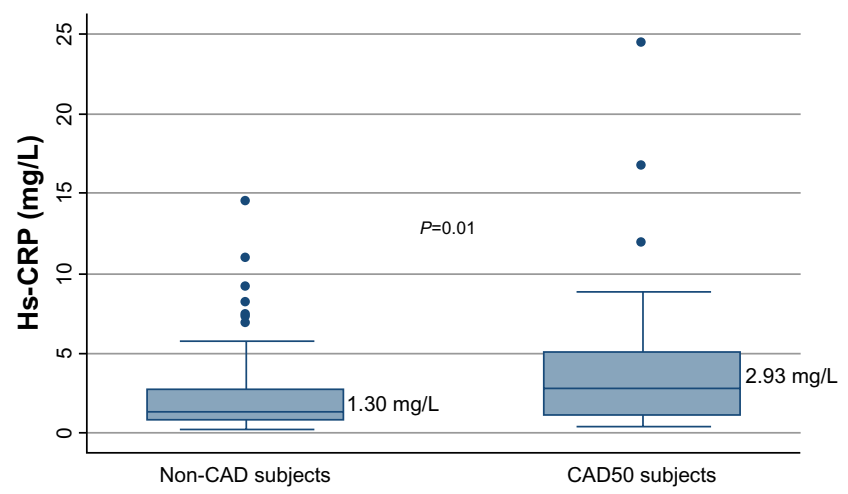

Figure 3 Box plots illustrating baseline hs-CRP in non-CAD subjects and CAD50 subjects.

Note: CAD50 refers to subjects with $\geq 50 \%$ coronary artery lumen diameter stenosis.

Abbreviations: hs-CRP, high-sensitivity C-reactive protein; CAD, coronary artery disease.
From baseline to 20 hours after peak exercise, no increase in hs-CRP was found with a delta hs-CRP of $-0.04 \mathrm{mg} / \mathrm{L}$ (IQR -0.30 to $0.18 \mathrm{mg} / \mathrm{L}$ ), $P=0.384$.

\section{Diagnostic performance of the ET, hs-CRP, and their combination}

For the diagnosis of CAD50, the AUC for the combined variable (positive if the ET was positive and/or baseline hs-CRP $>2.93 \mathrm{mg} / \mathrm{L}$ ) was not significantly larger compared to the AUC for the ET alone, 0.632 and 0.601 , respectively, $P=0.404$. Neither was the combined variable found to be superior to the

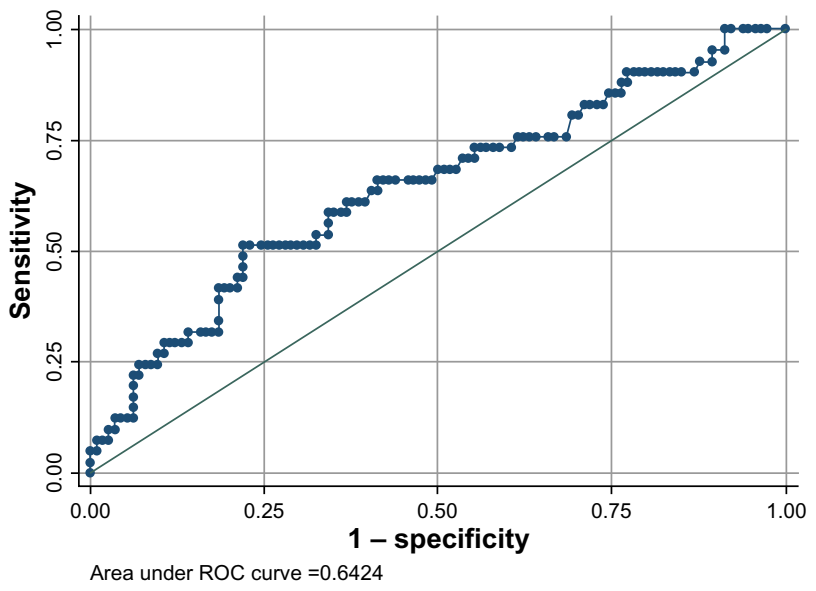

Figure 4 Receiver operating curve illustrating baseline hs-CRP and CAD50. Note: CAD50 refers to subjects with $\geq 50 \%$ coronary artery lumen diameter stenosis.

Abbreviations: ROC, receiver operating characteristic; hs-CRP, high-sensitivity C-reactive protein; CAD, coronary artery disease. 
Table 4 Multivariate logistic regression analysis with CAD50 as dependent variable

\begin{tabular}{llll}
\hline & \multicolumn{2}{l}{ Outcome CAD50 } & \\
\cline { 2 - 4 } & Odds ratio & $\mathbf{9 5 \%} \mathbf{~ C l}$ & P-value \\
\hline Hs-CRP & 2.32 & $0.95-5.69$ & 0.065 \\
Age & 1.10 & $1.05-1.15$ & $<0.001$ \\
Male sex & 5.96 & $2.11-16.83$ & 0.001 \\
Diabetes & 3.97 & $1.19-13.30$ & 0.025 \\
\hline
\end{tabular}

Notes: $\mathrm{Hs}$-CRP was dichotomized at $2.93 \mathrm{mg} / \mathrm{L}$. Cholesterol, estimated glomerular filtration rate, cigarette smoking, BMI, waist circumference, waist-hip ratio, daily physical activity level, systolic blood pressure, left ventricular ejection fraction, and ventricular hypertrophy were tested in the model but excluded because of $P$-value $>0.1$.

Abbreviations: $C A D$, coronary artery disease; hs-CRP, high-sensitivity C-reactive protein; $\mathrm{Cl}$, confidence interval; $\mathrm{BMI}$, body mass index.

ET alone in the diagnosis of CAD70 with an $\mathrm{AUC}=0.676$ compared to 0.685 for the ET, $P=0.837$.

\section{Discussion}

In this study, we found hs-CRP at rest to be positively associated with presence of CAD in univariate analysis, but after adjustment for age, sex, and diabetes, hs-CRP proved only borderline significant. Adding measurement of hs-CRP to the results of the ET did not improve the diagnostic evaluation of stable chest pain subjects in this study. We found hs-CRP to be a marker of CAD, but the clinical usefulness of the protein as a noninvasive diagnostic tool in the evaluation of ambulatory chest pain subjects was found to be limited by the fact that the marker was highly influenced by known cardiovascular risk factors.

We found hs-CRP to increase significantly after a symptom-limited ET, but the increase was not associated with presence of CAD. The exercise-induced increase in hs-CRP was modest with a median increase of $0.13 \mathrm{mg} / \mathrm{L}$ and was not associated with ET parameters or demographic data and was without clinical relevance. However, the results indicate that exercise has potential to cause unwanted variations in hs-CRP, which can affect results in epidemiological studies of ambulatory subjects. If a precise hs-CRP measurement with the least possible variations due to transient influences is wanted, it must be recommended that the study subjects are not exercising prior to blood samples.

We found baseline hs-CRP to be negatively correlated with daily life activity level, indicating that regular exercise reduces general inflammatory status. This is in accordance with results from a study by Kamal and Ragy examining obese children in which they found reduced CRP levels after 12 weeks exercise program. ${ }^{22} \mathrm{~A}$ study by Donges et al examining 102 sedentary subjects demonstrated that resistance training over a period of 10 weeks significantly reduced the CRP concentrations by $32.8 \%{ }^{23}$ The anti-inflammatory effects of regular exercise are proposed to be caused by a reduction in visceral fat and/or by induction of anti-inflammatory cytokines. ${ }^{24}$ That hypothesis is in accordance with our study reporting that hs-CRP levels are positively associated with BMI.

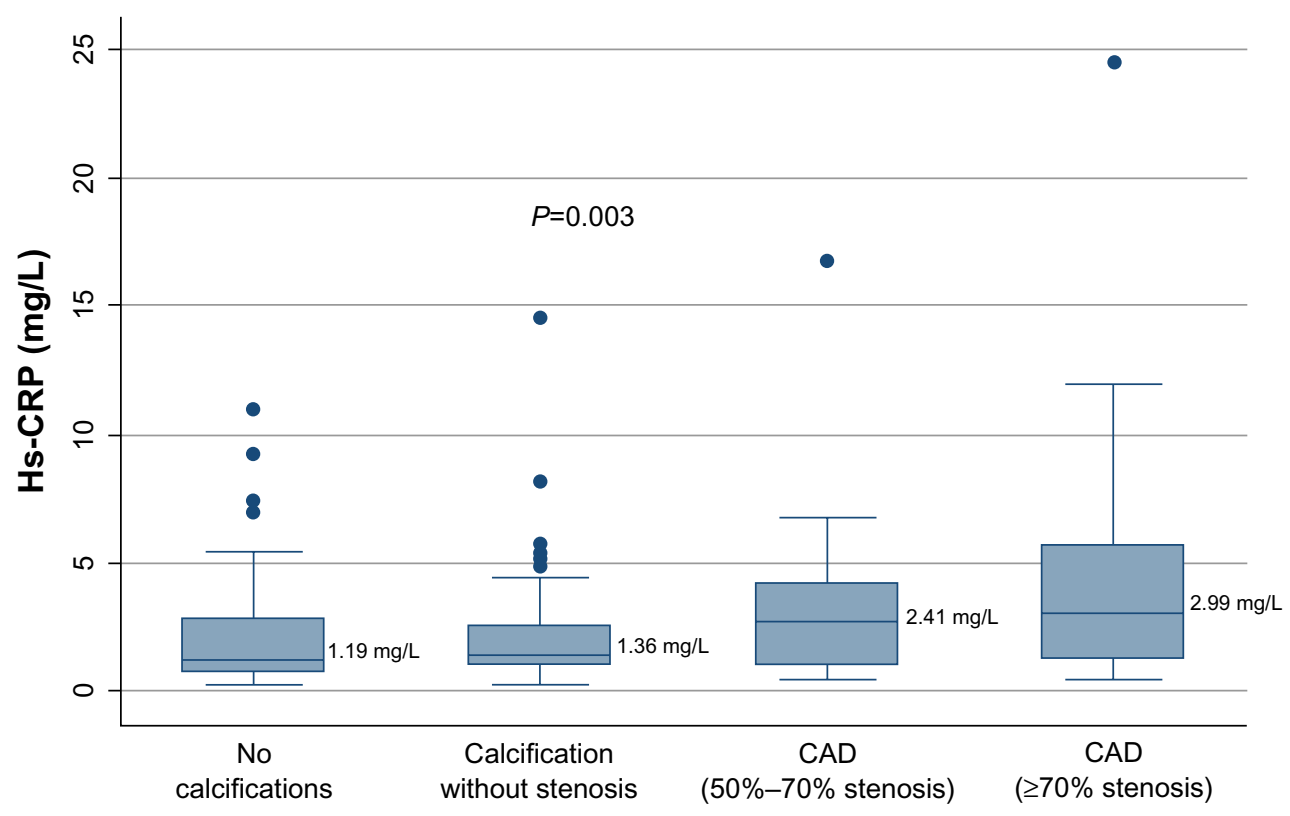

Figure 5 Median hs-CRP values in study subjects grouped in four groups.

Notes: The four groups are: no calcification in coronary arteries ( $n=64)$; calcifications without stenosis ( $n=50)$; CAD with $50 \%-70 \%$ stenosis $(n=16)$; and CAD with $\geq 70 \%$ stenosis $(n=25)$.

Abbreviations: hs-CRP, high-sensitivity C-reactive protein; CAD, coronary artery disease. 
Furthermore, we found baseline hs-CRP to be independently associated with baseline hs-TnT. Cardiac troponins are specific markers of myocyte necrosis, which is widely used in the diagnosis of acute myocardial infarction..$^{25}$ In recent years, the development of new and more sensitive assays has led to the intriguing discovery that healthy subjects without acute myocardial damage actually have circulating troponin in varying concentrations in their circulation. Data are now available documenting that increased hs-TnT levels, below the 99th percentile of a healthy population, are associated with adverse cardiovascular outcome. ${ }^{26}$ It is possible that hs-CRP and hs-TnT reflect a chronic state of low-grade inflammation and minor myocardial damage with joined origin.

Several studies have reported increased hs-CRP levels in subjects with acute myocardial infarction..$^{27,28}$ These findings explain and justify the clinical beneficial effects of statin therapy initiated immediately after an acute myocardial infarction. Whether hs-CRP increases as a response to reversible myocardial ischemia is still uncertain. In this study, we were not able to demonstrate an association between the release of hs-CRP in relation to exercise and the presence of CAD. Neither was increase in hs-CRP found to be related to ST segment depression or chest pain during the ET. A study supporting our results is a study by Başkurt et al where they found a slight - although nonsignificant - rise in serum hs-CRP 15 minutes after an ET, and the rise was not associated with the occurrence of reversible myocardial ischemia. ${ }^{29}$ Regarding baseline hs-CRP and reversible ischemia, a similar lack of correlation between baseline hs-CRP and reversible ischemia is reported from several studies. Shehadeh et al reported that there was no association between baseline hs-CRP and transient myocardial ischemia in patients with a history of chronic heart failure or previous myocardial infarction. ${ }^{30}$ Veselka et al could not find any relationship between hs-CRP levels and ET results in a study of 200 patients with positive ET. ${ }^{31}$ The only study available reporting a correlation between hs-CRP and reversible ischemia is a study by Cosin-Sales et al where they examined patients with chest pain and normal coronary angiography and found a correlation between hs-CRP and ST segment depression during ET..$^{32}$ These results, together with results from our study, suggest that hs-CRP is an indicator of a state of chronic systemic inflammation that predisposes to atherosclerosis, but that hs-CRP is not a marker of reversible ischemia. The reason for this apparent conflicting statement could be explained by the fact that CAD can be present without ischemic heart disease, and ischemic heart disease can be present without visible CAD. ${ }^{33}$

\section{The inflammatory hypothesis of atherothrombosis}

A study by Suzuki et al found a positive correlation between systemic CRP levels and coronary plaque area, suggesting an important connection between systemic level of inflammation and the extent of CAD. ${ }^{34}$ In our study, we also found an association between hs-CRP and Agatston score, but the association disappeared when an adjustment for the conventional risk factors was made. The fact that CRP interacts with several known cardiovascular risk factors has been one of the major problems regarding the incorporation of CRP into existing risk models. However, CRP has, in many trials, proved to be the most independent inflammatory marker. Within the Physicians' Health Study cohort, hs-CRP levels were better predictors of vascular events than several other inflammatory biomarkers, including fibrinogen, soluble intercellular adhesion molecule-1, soluble vascular adhesion molecule-1, serum amyloid A, and interleukin- $6 .{ }^{35}$ The observation made in that study that hs-CRP levels were associated with stroke risk also proved important with regard to understanding interactions between lipid-lowering therapy and inflammation, since statin therapy was known to reduce the risk of stroke, despite LDL cholesterol not being a major risk factor for stroke events. A considerable body of evidence now exists, suggesting that inflammation plays a major role in the initiation and progression of atherosclerosis and also in the development of unstable plaques in the coronary arteries. Whether CRP is a risk factor in itself or whether it is just a marker (an innocent bystander) of underlying generalized atherosclerosis is debatable. As an exquisitely sensitive marker of inflammation, CRP differences observed in individuals at higher risk of CAD may be reflecting the inflammatory activity of the atheroma rather than contributing to its development or progression. It is suggested that CRP acts as a marker for interleukin-6, a cellular cytokine associated with the recruitment of macrophages and monocytes into atherosclerotic plaques. In addition, CRP plays a role in the expression of tissue factor, a membrane glycoprotein that promotes coagulation. ${ }^{36}$

The role of hs-CRP as a prognostic marker of cardiovascular risk is well-established, but the role of CRP as a diagnostic marker of CAD is probably limited.

\section{Limitations}

Blood samples were obtained before, 5 minutes after, and 20 hours after peak exercise, and it is possible that the timing for obtaining the blood was not optimal for the purpose of detecting peak values in hs-CRP caused by 
reversible ischemia. Baseline hs-CRP was only measured once and subjects with brief and transient elevations of hs-CRP, for example due to minor subclinical infection, are therefore not distinguished from subjects with chronic elevation of hs-CRP. We found the association between hs-CRP and CAD to be borderline significant in multivariate analyses and it is possible that this study is underpowered in order to illustrate a true independent association.

\section{Conclusion}

Baseline hs-CRP was associated with presence of CAD, but when including conventional risk factors, the association was reduced to be borderline significant. Measuring hs-CRP did not improve the diagnostic evaluation of the stable chest pain subjects. Baseline hs-CRP was independently associated with BMI and hs-TnT and negatively associated with daily life activity level. Hs-CRP increased immediately as a response to exercise and returned to baseline levels 20 hours after exercise. The increase was modest, without clinical relevance, and not associated with CAD, but the effect of exercise should be taken into account when measuring hs-CRP in epidemiological studies to avoid unwanted variations.

\section{Acknowledgment}

This study was supported by the following foundations; Danish Heart Foundation (10-04-R79-A2930-22577). Th Maigaards Eftf. Fru Lily Benthine Lunds Fond, Direktoer Kurt Boennelycke og hustru Fru Grethe Boennelyckes Fond, Fru Gudrun Elboth mindelegat, Jens Anker Andersen Fonden, Henry og Astrid Moellers Fond, Grosserer Chr. Andersen og hustru Ingeborg Ovidia Signe Andersen Legat, Beckett Fonden, Snedkermester Sophus Jacobsen og hustru Astrid Jacobsens Fond, Krista og Viggo Petersens Fond, Alfred Helsteds og Eli Moellers legat, Murermester Laurits Peter Christensen og hustru Kirsten Sigrid Christensens Fond, Arvid Nielssons Fond, Else og Mogens Wedell-Wedellsborg Fond, and Axel Muusfeldts Fond.

\section{Disclosure}

The authors report no conflicts of interest in this work.

\section{References}

1. O’Donnell CJ, Elosua R. [Cardiovascular risk factors. Insights from Framingham Heart Study]. Rev Esp Cardiol. 2008;61(3):299-310. Spanish [with English abstract].

2. Doyle JT, Dawber TR, Kannel WB, Heslin AS, Kahn HA. Cigarette smoking and coronary heart disease. Combined experience of the Albany and Framingham studies. N Engl J Med. 1962;266:796-801.

3. Fox CS, Coady S, Sorlie PD, et al. Trends in cardiovascular complications of diabetes. JAMA. 2004;292(20):2495-2499.
4. Mora S, Cook N, Buring JE, Ridker PM, Lee IM. Physical activity and reduced risk of cardiovascular events: potential mediating mechanisms. Circulation. 2007;116(19):2110-2118.

5. Morris JN, Heady JA, Raffle PA, Roberts CG, Parks JW. Coronary heart-disease and physical activity of work. Lancet. 1953;265(6796): $1111-1120$.

6. Rosenberg L, Kaufman DW, Helmrich SP, Shapiro S. The risk of myocardial infarction after quitting smoking in men under 55 years of age. N Engl J Med. 1985;313(24):1511-1514.

7. Greenland P, Knoll MD, Stamler J, et al. Major risk factors as antecedents of fatal and nonfatal coronary heart disease events. JAMA. 2003;290(7):891-897.

8. Khot UN, Khot MB, Bajzer CT, et al. Prevalence of conventional risk factors in patients with coronary heart disease. JAMA. 2003;290(7): 898-904.

9. Magnus P, Beaglehole R. The real contribution of the major risk factors to the coronary epidemics: time to end the "only-50\%" myth. Arch Intern Med. 2001;161(22):2657-2660.

10. Ridker PM, Danielson E, Fonseca FA, et al. Rosuvastatin to prevent vascular events in men and women with elevated C-reactive protein. N Engl J Med. 2008;359(21):2195-2207.

11. Amarenco P, Goldstein LB, Szarek M, et al; SPARCL Investigators. Effects of intense low-density lipoprotein cholesterol reduction in patients with stroke or transient ischemic attack: the Stroke Prevention by Aggressive Reduction in Cholesterol Levels (SPARCL) trial. Stroke. 2007;38(12):3198-3204.

12. Fellström BC, Jardine AG, Schmieder RE, et al; AURORA Study Group. Rosuvastatin and cardiovascular events in patients undergoing hemodialysis. N Engl J Med. 2009;360(14):1395-1407.

13. Reslan OM, Khalil RA. Vascular effects of estrogenic menopausal hormone therapy. Rev Recent Clin Trials. 2012;7(1):47-70.

14. Nordestgaard BG, Zacho J. Lipids, atherosclerosis and CVD risk: is CRP an innocent bystander? Nutr Metab Cardiovasc Dis. 2009;19(8): 521-524.

15. Geluk CA, Post WJ, Hillege HL, et al. C-reactive protein and angiographic characteristics of stable and unstable coronary artery disease: data from the prospective PREVEND cohort. Atherosclerosis. 2008;196(1):372-382.

16. Nabata A, Kuroki M, Ueba H, et al. C-reactive protein induces endothelial cell apoptosis and matrix metalloproteinase-9 production in human mononuclear cells: Implications for the destabilization of atherosclerotic plaque. Atherosclerosis. 2008;196(1):129-135.

17. Fox K, Garcia MA, Ardissino D, et al; Task Force on the Management of Stable Angina Pectoris of the European Society of Cardiology; ESC Committee for Practice Guidelines. Guidelines on the management of stable angina pectoris: executive summary: The Task Force on the Management of Stable Angina Pectoris of the European Society of Cardiology. Eur Heart J. 2006;27(11):1341-1381.

18. Diamond GA, Forrester JS. Analysis of probability as an aid in the clinical diagnosis of coronary-artery disease. N Engl J Med. 1979;300(24): $1350-1358$.

19. Genders TS, Steyerberg EW, Alkadhi H, et al; CAD Consortium. A clinical prediction rule for the diagnosis of coronary artery disease: validation, updating, and extension. Eur Heart J. 2011;32(11): 1316-1330.

20. Gibbons RJ, Balady GJ, Beasley JW, et al. ACC/AHA Guidelines for Exercise Testing. A report of the American College of Cardiology/American Heart Association Task Force on Practice Guidelines (Committee on Exercise Testing). J Am Coll Cardiol. 1997;30(1):260-311.

21. Austen WG, Edwards JE, Frye RL, et al. A reporting system on patients evaluated for coronary artery disease. Report of the Ad Hoc Committee for Grading of Coronary Artery Disease, Council on Cardiovascular Surgery, American Heart Association. Circulation. 1975;51(Suppl 4):5-40.

22. Kamal NN, Ragy MM. The effects of exercise on C-reactive protein, insulin, leptin and some cardiometabolic risk factors in Egyptian children with or without metabolic syndrome. Diabetol Metab Syndr. 2012;4(1):27. 
23. Donges CE, Duffield R, Drinkwater EJ. Effects of resistance or aerobic exercise training on interleukin-6, C-reactive protein, and body composition. Med Sci Sports Exerc. 2010;42(2):304-313.

24. Astrom MB, Feigh M, Pedersen BK. Persistent low-grade inflammation and regular exercise. Front Biosci (Schol Ed). 2010;2:96-105.

25. Alpert JS, Thygesen K, Jaffe A, White HD. The universal definition of myocardial infarction: a consensus document: ischaemic heart disease. Heart. 2008;94(10):1335-1341.

26. Otsuka T, Kawada T, Ibuki C, Seino Y. Association between highsensitivity cardiac troponin $\mathrm{T}$ levels and the predicted cardiovascular risk in middle-aged men without overt cardiovascular disease. Am Heart J. 2010;159(6):972-978.

27. Auer J, Berent R, Lassnig E, Eber B. C-reactive protein and coronary artery disease. Jpn Heart J. 2002;43(6):607-619.

28. Blancke F, Claeys MJ, Jorens P, et al. Systemic inflammation and reperfusion injury in patients with acute myocardial infarction. Mediators Inflamm. 2005;2005(6):385-389.

29. Başkurt M, Aktürk F, Keskin K, et al. Serum high-sensitivity C-reactive protein, amyloid associated protein and N-terminal proBNP levels do not predict reversible myocardial ischaemia. Cardiovasc J Afr. 2011;22(2):85-89.
30. Shehadeh J, Lewis BS, Weisz G, David M, Ashkenazi T, Halon DA Relation between C-reactive protein, treadmill exercise testing, and inducible myocardial ischemia. Am J Cardiol. 2004;93(5):614-617.

31. Veselka J, Procházková S, Duchonová R, et al. Relationship of C-reactive protein to presence and severity of coronary atherosclerosis in patients with stable angina pectoris or a pathological exercise test. Coron Artery Dis. 2002;13(3):151-154.

32. Cosin-Sales J, Pizzi C, Brown S, Kaski JC. C-reactive protein, clinical presentation, and ischemic activity in patients with chest pain and normal coronary angiograms. J Am Coll Cardiol. 2003;41(9):1468-1474.

33. Marzilli M, Merz CN, Boden WE, et al. Obstructive coronary atherosclerosis and ischemic heart disease: an elusive link! J Am Coll Cardiol. 2012;60(11):951-956.

34. Suzuki M, Saito M, Nagai T, Saeki H, Kazatani Y. Systemic versus coronary levels of inflammation in acute coronary syndromes. Angiology. 2006;57(4):459-463.

35. Ridker PM, Brown NJ, Vaughan DE, Harrison DG, Mehta JL. Established and emerging plasma biomarkers in the prediction of first atherothrombotic events. Circulation. 2004;109(25 Suppl 1):IV6-IV19.

36. Hansson GK. Inflammation, atherosclerosis, and coronary artery disease. $N$ Engl J Med. 2005;352(16):1685-1695.

\section{Publish your work in this journal}

The Journal of Inflammation Research is an international, peer-reviewed open-access journal that welcomes laboratory and clinical findings on the molecular basis, cell biology and pharmacology of inflammation including original research, reviews, symposium reports, hypothesis formation and commentaries on: acute/chronic inflammation; mediators of inflamma-

\section{Dovepress}

tion; cellular processes; molecular mechanisms; pharmacology and novel anti-inflammatory drugs; clinical conditions involving inflammation. The manuscript management system is completely online and includes a very quick and fair peer-review system. Visit http://www.dovepress.com/ testimonials.php to read real quotes from published authors. 